\title{
Histone deacetylase inhibitors valproic acid and depsipeptide sensitize retinoblastoma cells to radiotherapy by increasing H2AX phosphorylation and p53 acetylation-phosphorylation
}

\author{
TAKESHI KAWANO ${ }^{1}$, MASAHARU AKIYAMA ${ }^{1,2}$, MIYUKI AGAWA-OHTA ${ }^{1}$, YOKO MIKAMI-TERAO ${ }^{1,2}$, \\ SATSUKI IWASE ${ }^{1}$, TAKAAKI YANAGISAWA ${ }^{3}$, HIROYUKI IDA $^{2}$, NAOKI AGATA $^{1}$ and HISASHI YAMADA ${ }^{1}$ \\ ${ }^{1}$ Division of Molecular Genetics, Institute of DNA Medicine, and ${ }^{2}$ Department of Pediatrics, Jikei University School \\ of Medicine, Tokyo 105-8461; ${ }^{3}$ Division of Pediatric Neuro-Oncology, Department of Neuro-Oncology, \\ Saitama Medical University International Medical Center, Saitama 350-1298, Japan
}

Received February 16, 2010; Accepted March 18, 2010

DOI: 10.3892/ijo_00000728

\begin{abstract}
Although p53 is intact in most cases of retinoblastoma, it is largely inactivated by the ubiqutin-proteasome system through interaction with murine double minute 2 (MDM2) and murine double minute X (MDMX). The present study showed that the histone deacetylase (HDAC) inhibitors valproic acid (VPA) and depsipeptide (FK228) synergistically enhanced ionizing radiation (IR)-induced apoptosis, associated with activation of caspase-3 and cleavage of poly(ADP-ribose) polymerase in Y79 and WER1-Rb1 human retinoblastoma cells. Both VPA and FK228 enhanced IR-induced phosphorylation of histone H2AX on Ser139 preceding apoptosis. Exposure of cells to IR in the presence of VPA or FK228 induced the accumulation of p53 acetylated at Lys382 and phosphorylated at Ser46 through the reduction of binding affinity with MDM2 and MDMX. These results suggest that acetylation of p53 by HDAC inhibitors is a promising new therapeutic target in refractory retinoblastoma.
\end{abstract}

\section{Introduction}

Retinoblastoma is the most common malignant intraocular tumor in childhood. Over the last 20 years, molecular biology and genetics studies have described the tumorigenic characteristics of retinoblastoma $(1,2)$. This tumor arises from a germline or somatic mutation at the retinoblastoma 1 (RB1) gene on chromosome $13 \mathrm{q}$. Current treatments for this tumor include systemic chemotherapy and focal conservative treatments, such as chemotherapy, thermotherapy, and cryotherapy $(2,3)$. These therapies have significantly improved the survival rate and the visual-function salvage rate of patients with

Correspondence to: Dr Masaharu Akiyama, Department of Pediatrics, Jikei University School of Medicine, 3-25-8 Nishishinbashi, Minato-ku, Tokyo 105-8461, Japan

E-mail: makiyama@jikei.ac.jp

Key words: retinoblastoma, histone deacetylase inhibitor, p53 retinoblastoma. Although such treatments as enucleation and external beam radiotherapy are avoided because of the risks of orbital dysgenesis and secondary neoplasm induction, external beam radiotherapy remains indicated for cases refractory to systemic chemotherapy or focal treatments. Therefore, to treat retinoblastoma refractory to conventional therapy a safer therapeutic approach, which would allow lower doses of radiation to preserve visual function and to avoid radiationrelated side effects, including secondary neoplasm, is needed.

The p53 gene is deleted or mutated and non-functional in many human cancers but is intact and functional in most cases of retinoblastoma (4-6). Although p53 is usually intact in retinoblastoma, it is inactivated by murine double minute 2 (MDM2) and murine double minute X (MDMX), also known as MDM4, through the ubiqutin-proteasome degradation system (7-11). Histone deacetylase (HDAC) inhibitors exhibit antitumor effects by acetylating chromosomal protein histones and transcriptional factors, including p53 (12). When acetylated, p53 accumulates because it is no longer subject to ubiqutination by MDM2/MDMX and subsequent proteasomal degradation (8-11). Thus, HDAC inhibitors might be able to restore the p53 pathway and to induce p53-mediated cell cycle arrest and cell death in retinoblastoma cells. Recent studies have shown that valproic acid (VPA, 2-propylpentanoic acid), which is used as an antiepileptic drug and mood stabilizer, has antitumor activity through inhibition of HDACs $(13,14)$. Moreover, FK228, also known as depsipeptide, is a novel, natural, bicyclic tetrapeptide with antitumor activities, which are mostly mediated by inhibition of HDACs $(15,16)$. In this study, we investigated the effects and the molecular mechanisms of VPA and FK228 on ionizing radiation (IR)-induced apoptosis in retinoblastoma cells.

\section{Materials and methods}

Cell culture. The human retinoblastoma cell lines Y79 and WERI-Rb1 were obtained from the Riken Cell Bank (Tsukuba, Japan) and were cultured in RPMI-1640 (Mediatech, Herndon, VA, USA) with $10 \%$ fetal bovine serum (Hyclone, Logan, UT, USA), containing $2 \mathrm{mM}$ L-glutamine, $100 \mathrm{U} / \mathrm{ml}$ penicillin, and 
$100 \mu \mathrm{g} / \mathrm{ml}$ streptomycin (Wako Pure Chemical Industries, Ltd., Osaka, Japan) in a $5 \% \mathrm{CO}_{2}$ incubator with $100 \%$ humidity.

Reagents and irradiation. FK228 was kindly provided by Fujisawa Pharmaceutical Co. (Osaka, Japan). VPA was purchased from Sigma-Aldrich (St. Louis, MO, USA). All stock solutions were made in dimethylsulfoxide and stored at $-20^{\circ} \mathrm{C}$ until use. For irradiation, suspension cultures were exposed to single doses of 2, 5, or 10 Gy with an X-ray generator (MBR-1520R, Hitachi, Tokyo, Japan). Viable cells were counted with the trypan-blue dye exclusion method by means of a hematocytometer and a phase-contrast microscope. All experiments were performed in triplicate.

Analysis of apoptotic cells. Y79 and WERI-Rb1 cells were stained with propidium iodide (PI) and analyzed with flow cytometry to detect cells with sub-G1 DNA content. Cells were also stained with PI and Bisbenzimide Hoechst 33342 (Sigma-Aldrich) to identify apoptotic cells by examining nuclear fragmentation and cell-membrane permeability under a fluorescence microscope. Five hundred cells were scored for apoptosis in each of 3 independent experiments.

Immunoblotting. Cells were harvested, washed with ice-cold PBS, and lysed by sonication in the presence of buffer containing $50 \mathrm{mM}$ Tris- $\mathrm{HCl}$ (pH 7.4), $150 \mathrm{mM} \mathrm{NaCl}, 1 \%$ Nonidet P-40, $2 \mathrm{mM}$ sodium orthovanadate, and a protease inhibitor cocktail (Roche Diagnostics Corp., Indianapolis, IN, USA). An equal amount ( $30 \mu \mathrm{g})$ of the samples was separated on sodium dodecylsulfate-polyacrylamide gel and then transferred onto nitrocellulose filters (Bio-Rad Laboratories, Hercules, CA, USA). The membranes were immunoblotted with antibodies against caspase-3 (Cell Signaling Technology, Beverly, MA, USA), poly(ADP-ribose) polymerase (PARP, Cell Signaling Technology), B-actin (Santa Cruz Biotechnology Inc., Santa Cruz, CA, USA), MDM2 (Santa Cruz Biotechnology, Inc.), MDMX (Bethyl Laboratories, Inc., Montgomery, TX, USA), acetylated p53 (Lys382) (Cell Signaling Technology), p53 (Santa Cruz Biotechnology Inc.), and $\alpha$-tubulin (Sigma-Aldrich). The immunoblots were incubated with anti-mouse or anti-rabbit IgG-HRP antibodies (Santa Cruz Biotechnology, Inc.), and then were detected with enhanced chemiluminescence (ECL Plus, GE Health Care, Buckinghamshire, UK).

Immunoprecipitation. Whole-cell lysates (WCLs) were incubated with antibodies against p53 (Santa Cruz Biotechnology Inc.), phospho-p53 (Ser46) (Cell Signaling Technology), acetylated p53 (Lys382) (Cell Signaling Technology), MDM2 (Santa Cruz Biotechnology, Inc.) or MDMX (Bethyl Laboratories, Inc.), normal mouse or rabbit IgG (Santa Cruz Biotechnology, Inc.) for $2 \mathrm{~h}$ at $4^{\circ} \mathrm{C}$ and then immunoprecipitated for $4 \mathrm{~h}$ with a TrueBlot anti-Rabbit Ig IP Beads (eBioscience, San Diego, CA, USA) or protein A/G beads (Thermo Fisher Scientific, Inc., Waltham, MA, USA). Immune complexes were washed, electrophoresed, and analyzed with immunoblotting using antibodies against MDM2, MDMX, acetylated p53 (Lys382), phospho-p53 (Ser46), or p53. The immunoblots were incubated with a Rabbit TrueBlot: HRP IgG (eBioscience) or anti-mouse or anti-rabbit IgG-HRP
A

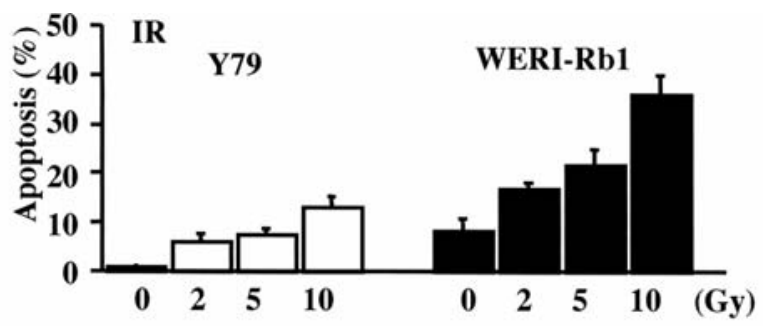

B $\frac{2}{\frac{2}{5}}$
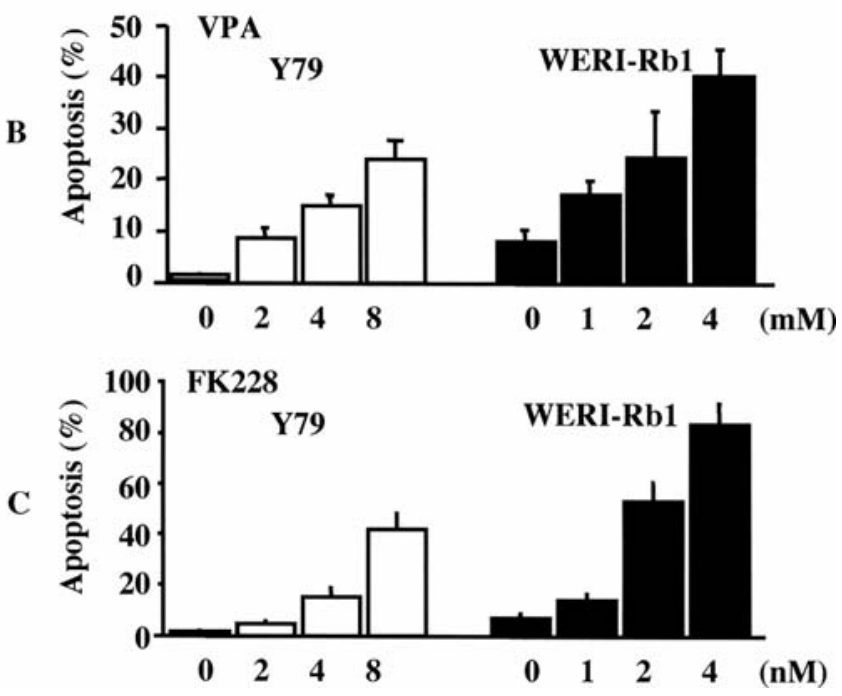

Figure 1. IR and HDAC inhibitors (VPA and FK228) induce apoptosis dosedependently in retinoblastoma cells. Y79 and WERI-Rb1 cells were treated with various doses of radiation (A), VPA (B), or FK228 (C). After 48 h, cells were harvested and stained with PI and Hoechst dye. Apoptotic cells were identified under a fluorescence microscope on the basis of fragmented nuclei and membrane permeability. Values represent the mean \pm SD of the results of 3 independent experiments.

antibodies (Santa Cruz Biotechnology, Inc.), and were then detected with ECL plus chemiluminescence (GE Health Care).

Immunofluorescence. The treated cells were washed with $10 \mathrm{mM}$ glycine in PBS and fixed with $2 \%$ paraformaldehyde at room temperature for $15 \mathrm{~min}$. Coverslips were treated with blocking solution ( $5 \%$ bovine serum albumin, $0.05 \%$ Tween-20 in PBS) for $1 \mathrm{~h}$. Cells were then incubated with antibodies against phospho-histone H2AX (Ser139) (Cell Signaling Technology) for $1 \mathrm{~h}$ at room temperature. After being washed with PBS-T, the coverslips were incubated with Alexa Fluor 488 anti-rabbit IgG (Invitrogen, Eugene, OR, USA) for $45 \mathrm{~min}$. Digital images were obtained with an Axioplan 2 microscope (Carl Zeiss Microimaging, Jena, Germany).

Statistical analysis. A paired t-test was performed with Stat View 4.5 software (Abacus Concepts, Inc., Berkeley, CA, USA). The minimal level of significance was $\mathrm{p}<0.05$.

\section{Results}

IR and HDAC inhibitors (VPA and FK228) induced apoptosis in retinoblastoma cells. We first examined the radiosensitivity of retinoblastoma cell lines. In both Y79 and WERI-Rb1 cells treated with IR, the rates of apoptosis after $48 \mathrm{~h}$ increased with the IR dose (0, 2, 5, and 10 Gy) (Fig. 1A). 


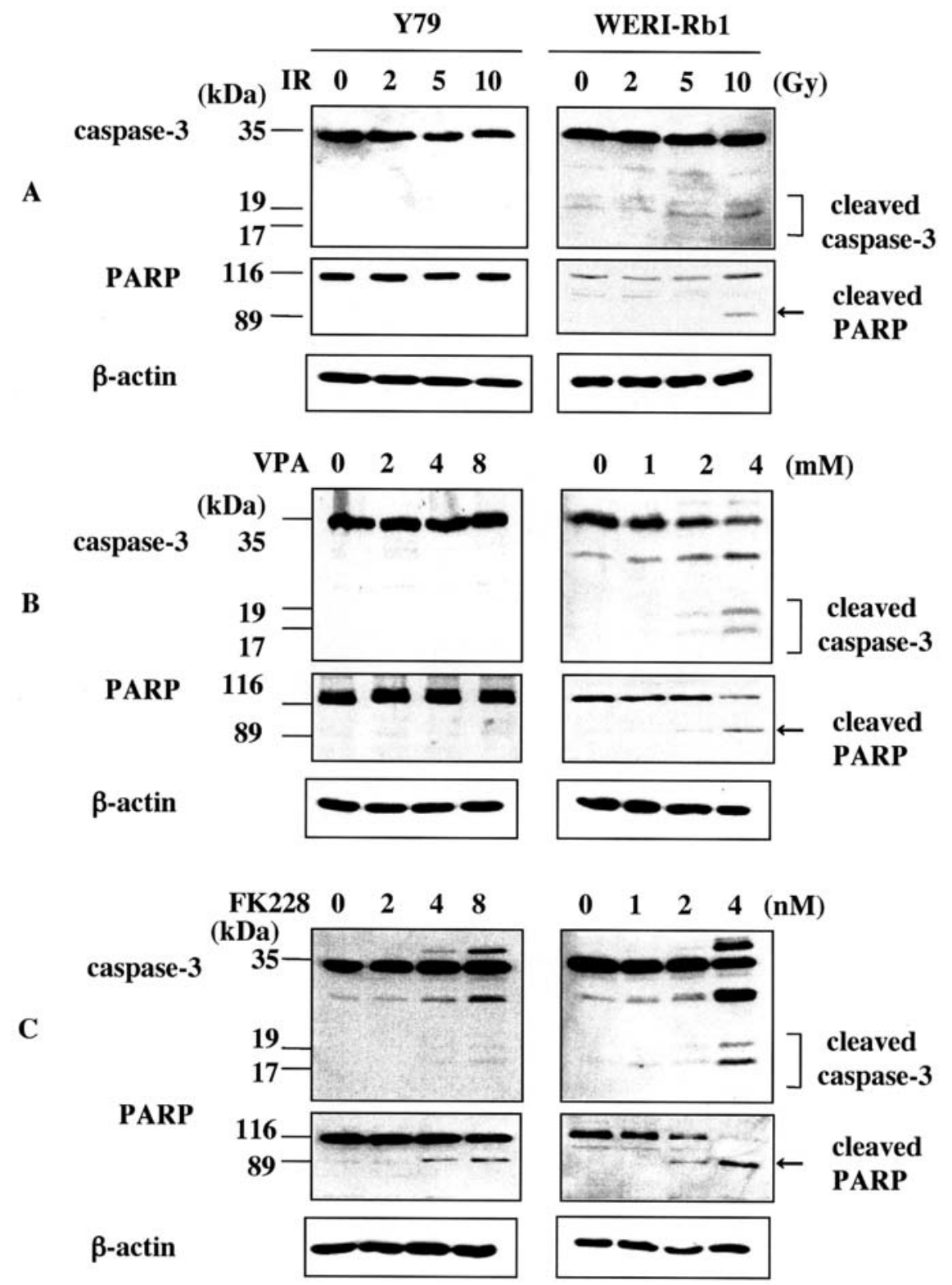

Figure 2. Induction of apoptosis induced by IR and HDAC inhibitors (VPA and FK228) was associated with cleaved caspase-3 and PARP in retinoblastoma cells. Samples treated with (A) IR $(0,2,5$, or $10 \mathrm{~Gy})$, (B) VPA $(0,2,4$, or $8 \mathrm{mM}$ in Y79 cells, and 0, 1, 2, or $4 \mathrm{mM}$ in WERI-Rb1 cells), and (C) FK228 (0, 2, 4 , or $8 \mathrm{nM}$ in Y79 cells, and 0, 1,2, or $4 \mathrm{nM}$ in WERI-Rb1 cells) for $48 \mathrm{~h}$ were immunoblotted for caspase-3 and PARP.

We next assessed the susceptibility of retinoblastoma cells to the HDAC inhibitors VPA (Y79 cells: $0,2,4$, and $8 \mathrm{mM}$; WERI-Rb1 cells: $0,1,2$, and $4 \mathrm{mM})$ and FK228 (Y79 cells: $0,2,4$, and $8 \mathrm{nM}$; WERI-Rb1 cells: $0,1,2$, and $4 \mathrm{nM}$ ). In both Y79 and WERI-Rb1 cells treated with VPA or FK228, the rates of apoptosis after $48 \mathrm{~h}$ increased in a dose-dependent manner (Fig. 1B and C).

In Y79 cells neither IR $(2,5$, or $10 \mathrm{~Gy})$ nor VPA $(2,4$, or $8 \mathrm{mM}$ ) induced caspase- 3 activation or PARP cleavage (Fig. 2A and B), whereas FK228 (4 or $8 \mathrm{nM}$ ) induced both caspase-3 activation and PARP cleavage (Fig. 2C). In WERI$\mathrm{Rb} 1$ cells, on the other hand, treatment with IR (10 Gy), VPA $(4 \mathrm{mM})$, or FK228 (4 or $8 \mathrm{nM})$ induced both caspase-3 activation and PARP cleavage (Fig. 2).

HDAC inhibitors synergistically enhanced IR-induced apoptosis in retinoblastoma cells. Both VPA and FK228 significantly enhanced IR-induced apoptosis in Y79 cells $(\mathrm{p}<0.01$; Fig. 3A). The rates of apoptosis with both FK228 or VPA and IR were greater than those with FK228 or VPA alone, which in turn were greater than those with IR alone. In Y79 cells the percentages of apoptosis were $7.9 \pm 3.3 \%$ with 2-Gy irradiation alone, $12.9 \% \pm 3.3 \%$ with VPA $(2 \mathrm{mM})$ alone, $46.9 \pm 14.4 \%$ with both 2 -Gy irradiation and VPA $(2 \mathrm{mM})$, $5.9 \pm 2.2 \%$ with FK228 (2 nM) alone, and $45.2 \pm 11.9 \%$ with both $2 \mathrm{~Gy}$ irradiation and FK228 $(2 \mathrm{nM})$.

Moreover, VPA and FK228 also significantly enhanced apoptosis in irradiated WERI-Rb1 cells $(\mathrm{p}<0.01)$ (Fig. 3A). The rates of apoptosis with both FK228 or VPA and IR were greater than those with FK228 or VPA alone, which in turn were greater than those with IR alone. The percentages of apoptotic cells were $12.0 \pm 2.4 \%$ with 2 -Gy irradiation, $17.0 \pm 2.7 \%$ with VPA $(1 \mathrm{mM}), 76.9 \pm 6.5 \%$ with both $2-\mathrm{Gy}$ irradiation and VPA $(1 \mathrm{mM}), 13.8 \pm 4.4 \%$ with FK228 $(1 \mathrm{nM})$, 
$\mathbf{A}$
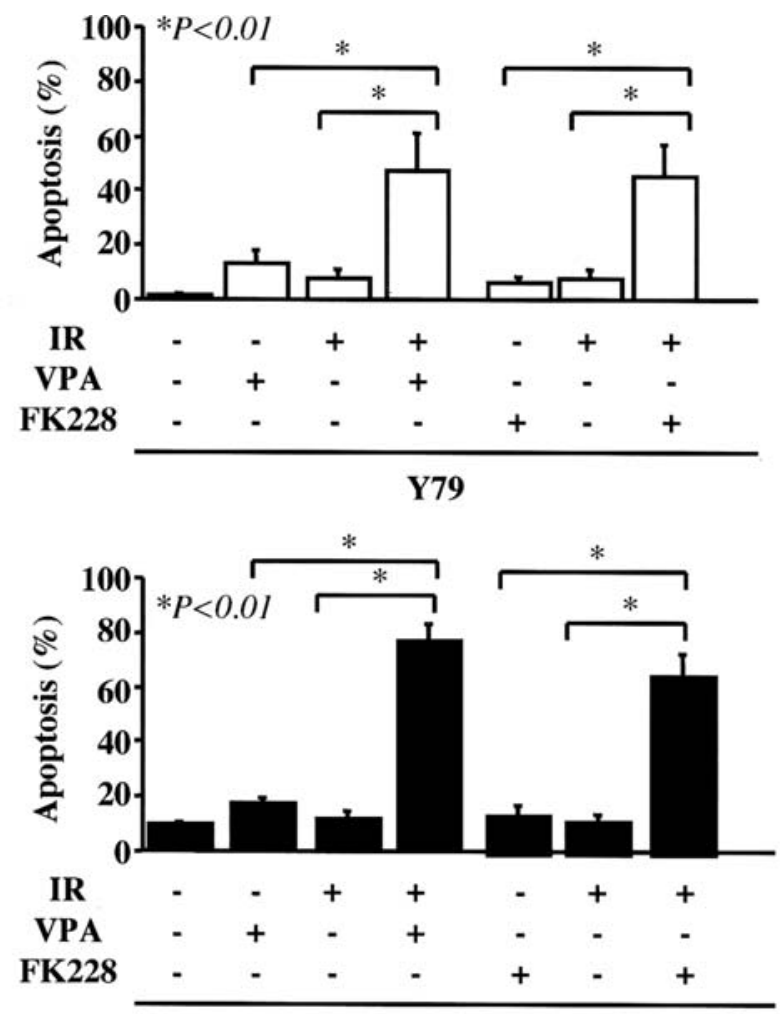

WERI-Rb1

B

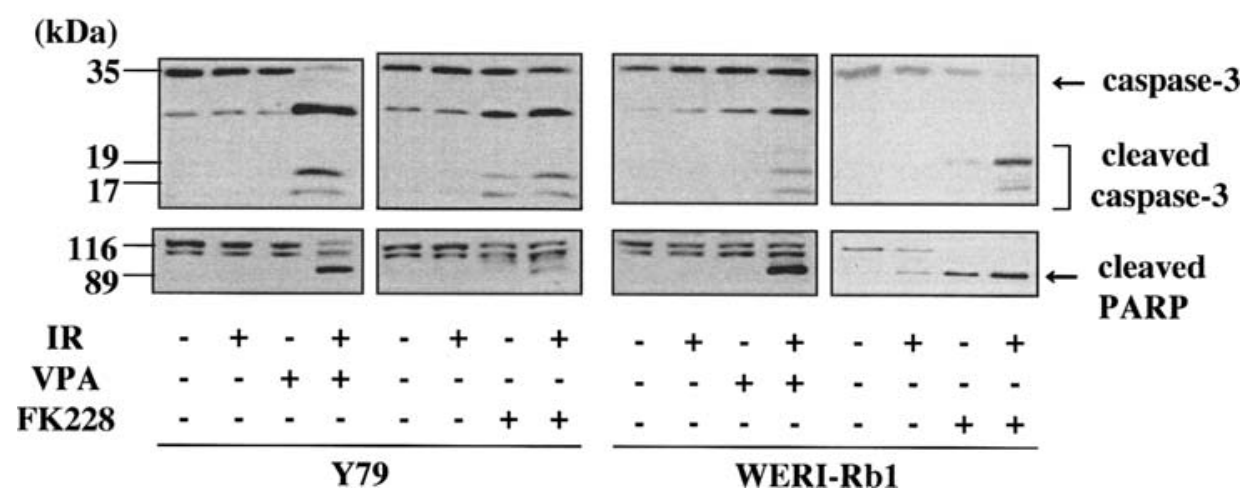

Figure 3. HDAC inhibitors enhanced IR-induced apoptosis, associated with cleaved caspase-3 and PARP, in retinoblastoma cells. (A) Y79 and WERI-Rb1 cells were treated with IR (2 Gy), VPA ( $2 \mathrm{mM}$ in Y79 cells, and $1 \mathrm{mM}$ in WERI-Rb1 cells) or FK228 (2 nM in Y79 cells, and $1 \mathrm{nM}$ in WERI-Rb1 cells) alone or in combination. After $48 \mathrm{~h}$, cells were harvested and stained with PI and Hoechst dye. Apoptotic cells were identified under a fluorescence microscope on the basis of fragmented nuclei and membrane permeability. Values represent the mean \pm SD of the results of 3 independent experiments. (B) Samples treated with IR (2 Gy), VPA (2 mM in Y79 cells, and $1 \mathrm{mM}$ in WERI-Rb1 cells), or FK228 ( $2 \mathrm{nM}$ in Y79 cells, and $1 \mathrm{nM}$ in WERI-Rb1 cells) alone, both IR and VPA, or both IR and FK228 were immunoblotted for caspase-3 and PARP.

and $65.2 \pm 8.1 \%$ with both 2-Gy irradiation and FK228 (1 nM). Enhancement of IR-induced apoptosis by VPA and FK228 was associated with the cleavage of both caspase- 3 and PARP (Fig. 3B).

HDAC inhibitors enhance IR-induced phosphorylation of histone H2AX in Y79 cells and WERI-Rb1 cells. To determine the molecular mechanism by which VPA and FK228 enhance IR-induced apoptosis, the effects of VPA and FK228 on the phosphorylation of histone H2AX on Ser139 was examined as a marker of DNA double-strand breaks (DSBs). Fluoresence microscopy revealed that IR (2 Gy) induced phosphorylation of H2AX in both Y79 and WERI-Rb1 cells (Fig. 4). VPA and FK228 enhanced the IR-induced phosphorylation of $\mathrm{H} 2 \mathrm{AX}$ in both Y79 and WERI-Rb1 cells, whereas each of these 2 agents alone induced nominal phosphorylation of H2AX (Fig. 4).

Effects of HDAC inhibitors on the expression of p53, acetylated p53 and MDM2/X in irradiated retinoblastoma cells. We evaluated the effects of HDAC inhibitors on the expression of p53, acetylated p53, and MDM2/X in irradiated Y79 and WERI-Rb1 cells. The expression of p53 was increased in WCLs from both cells treated with IR alone, both IR and VPA, or both IR and FK228 (Fig. 5). p53 was acetylated at Lys 382 by combination treatments with IR and VPA or with 


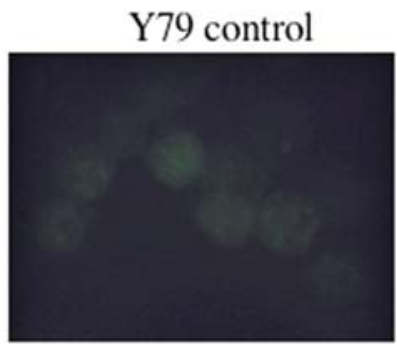

A
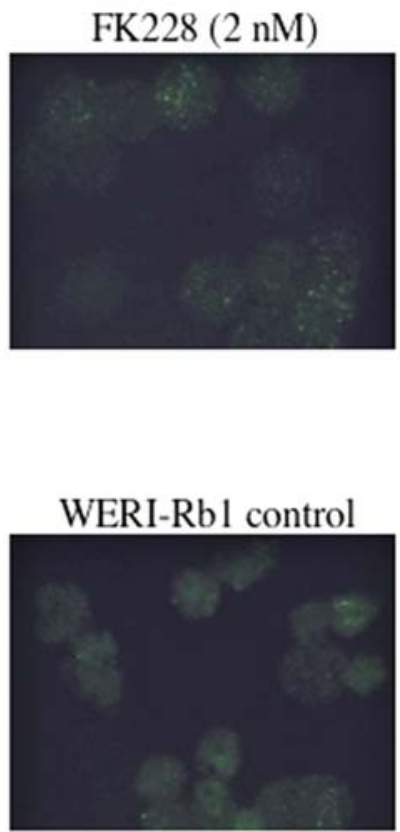

B

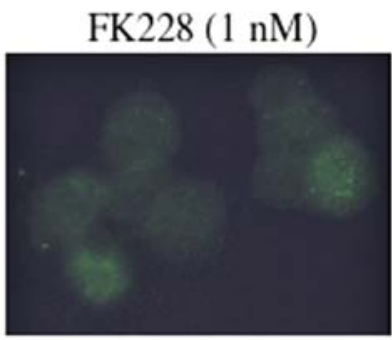

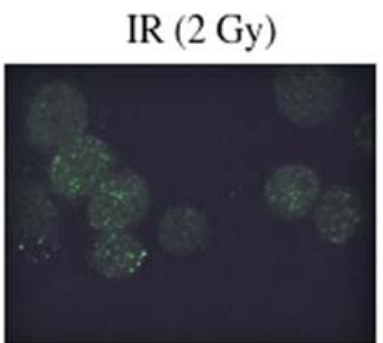
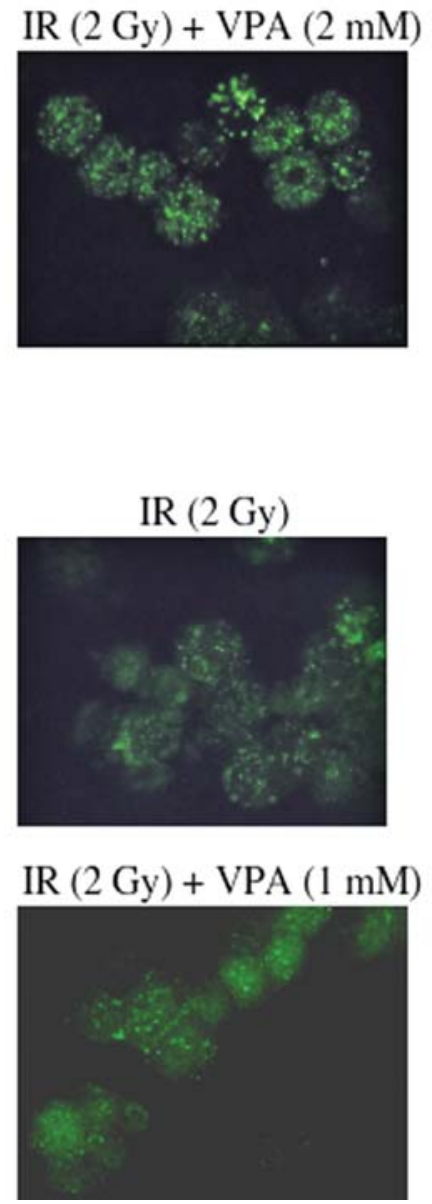
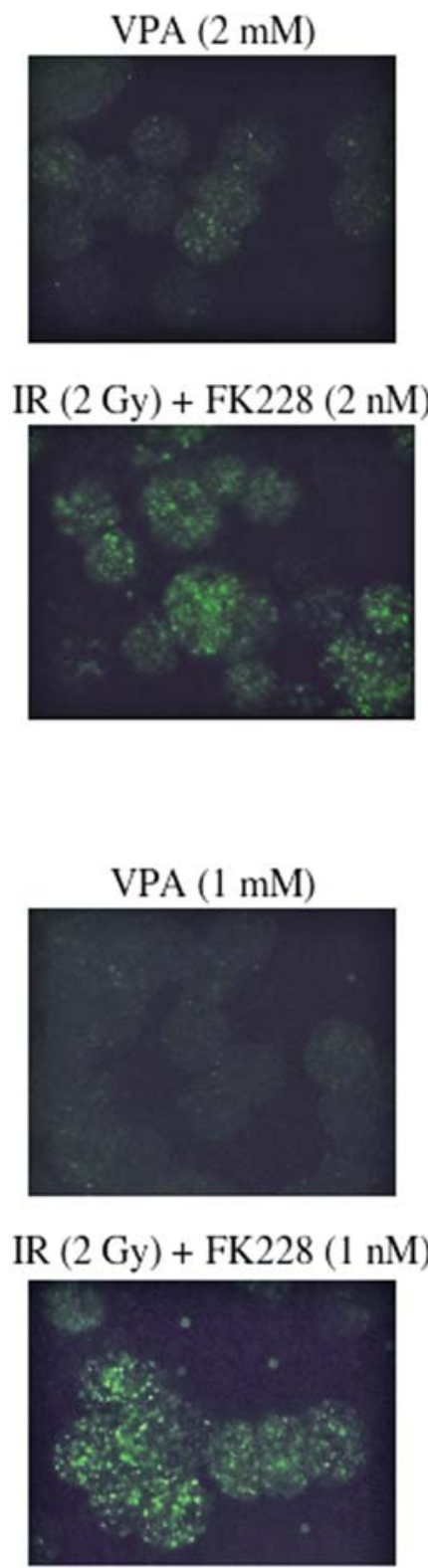

Figure 4. HDAC inhibitors enhanced IR-induced phosphorylation of histone H2AX in Y79 and WERI-Rb1 cells. Y79 cells (A) and WERI-Rb1 cells (B) were treated with IR, VPA, or FK228 alone, both IR and VPA, or both IR and FK228. Treated cells were incubated with an anti-phospho-histone H2AX (Ser139) antibody for $1 \mathrm{~h}$ at room temperature. After being washed with PBS-T, the coverslips were incubated with 488 Alex Fluor anti-rabbit IgG for 45 min. Digital images were obtained with an Axioplan 2 microscope.

IR and FK228, as well as by VPA or FK228 alone, in WCLs from both cell lines (Fig. 5). There were no changes in the expression of MDM2 or MDMX in WCLs from cells treated with IR, VPA, or FK228 alone, both IR and VPA, or both IR and FK228 (Fig. 5).

Effects of HDAC inhibitors on the interaction with p53 and $M D M 2 / X$ in irradiated retinoblastoma cells. We next investigated that effects of HDAC inhibitors on the interaction of p53 with MDM2/X. Immunoprecipitation (IP) assay with p53 antibody revealed that VPA and FK228 reduced the interaction of $\mathrm{p} 53$ and MDM2/X in both cells with or without irradiation (Fig. 5). Moreover, IP assay with MDM2/X antibodies showed that VPA and FK228 reduced the interaction of MDM2/X and p53 in both cell types with irradiation (Fig. 6).
Combination treatment with IR and VPA or with IR and FK228 markedly increased the acetylation of p53 in the samples immunoprecipitated with p53 antibody, as well as WCLs, from both cell lines (Fig. 5).

Effects of HDAC inhibitors on the acetylation-phosphorylation of p53 in irradiated retinoblastoma cells. The IP assay with phosphorylated p53 (Ser46) antibody showed that the acetylation at Lys382 of phosphorylated p53 (Ser46) was increased by VPA and FK228 in irradiated WERI-Rb1 cells but was not clearly increased in irradiated Y79 cells (Fig. 7). Conversely, IP assay with an acetylated p53 (Lys382) antibody revealed that VPA and FK228 increased the expression of p53 and phosphorylated p53 (Ser46) in irradiated Y79 and WERI-Rb1 cells (Fig. 7). 
Y79

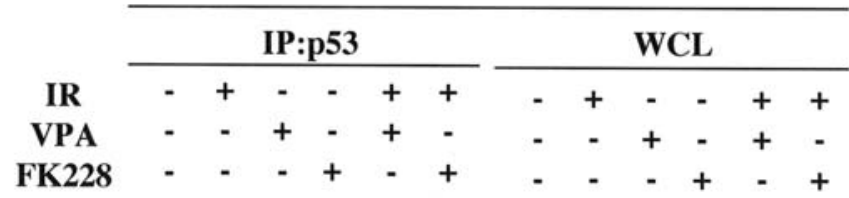

IB : MDM2
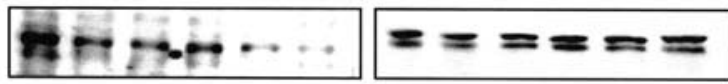

A

IB : MDMX

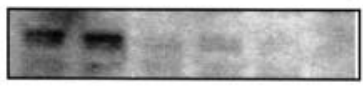

प्याओ

IB : Acetyl-p53 (Lys382)
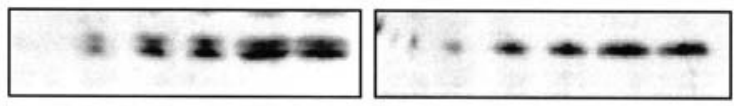

IB : p53

IB : $\alpha$-tubulin
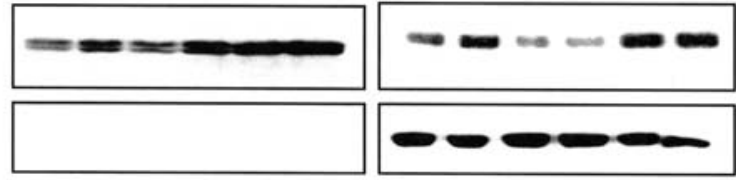

WERI-Rb1

\begin{tabular}{|c|c|c|c|c|c|c|c|c|c|c|}
\hline \multirow{3}{*}{$\begin{array}{c}\text { IR } \\
\text { VPA }\end{array}$} & \multicolumn{5}{|c|}{ IP:p53 } & \multicolumn{5}{|c|}{ WCL } \\
\hline &.- & & - & & + & & + & & & \\
\hline & - & + & - & + & - & - & - & + & + & - \\
\hline K228 & - & - & + & & + & - & - & -+ & - & + \\
\hline
\end{tabular}

IB : MDM2
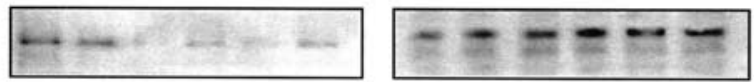

B

IB : MDMX
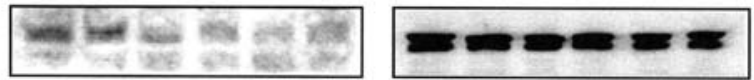

IB : Acetyl-p53 (Lys382)
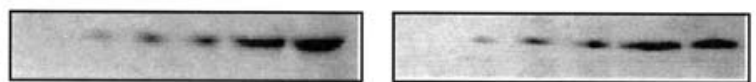

$$
\text { IB : p53 }
$$

IB : $\boldsymbol{\alpha}$-tubulin
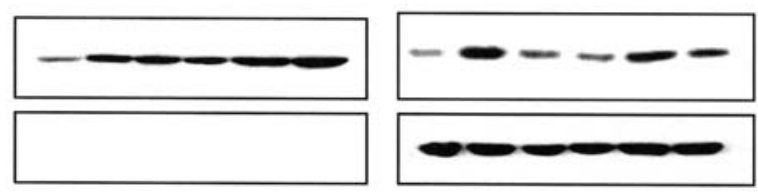

Figure 5. Effect of HDAC inhibitors on the expression and interaction of p53 and MDM2/X. Samples treated with both IR (2 Gy) and VPA (2 mM in Y79 cells, and $1 \mathrm{mM}$ in WERI-Rb1 cells) or both IR and FK228 ( $2 \mathrm{nM}$ in Y79 cells, and $1 \mathrm{nM}$ in WERI-Rb1 cells) for $48 \mathrm{~h}$ were immunoprecipitated for p53 and then immunoblotted for MDMX, MDM2, acetylated p53 (Lys382), and p53 in Y79 cells (A) and WERI-Rb1 cells (B). Moreover, WCL samples were immunoblotted for MDMX, MDM2, acetylated p53, p53, and $\alpha$-tubulin.

\section{Discussion}

The major finding of the present study is that the HDAC inhibitors VPA and FK228 sensitize retinoblastoma cells to IR, which is associated with phosphorylation of histone H2AX on Ser139 and accumulation of acetylated and phosphorylated p53. HDAC inhibitors, such as VPA, MS-275, m-carboxyciannamic acid bishydroxamide, and FK228, have been reported to enhance IR-induced apoptosis in K562 leukemic cells, U251 and T98G glioma cells, DU145 prostate cells, A549 pulmonary adenocarcinoma, and MKN45 gastric adenocarcinoma cells (17-19). The HDAC inhibitors trichostatin A, suberoylanilide hydroxamic acid (SAHA), and MS-275 have been shown to exert additive growth-inhibitory effects in combination with chemotherapeutic agents, such as carboplatin, etoposide, and vincristine, in retinoblastoma cells (20). However, the molecular mechanism by which HDAC inhibitors enhance sensitivity for genotoxic stress remain unknown.

IR induces DSBs in DNA, and histone H2AX is a subunit of the nucleosome that is rapidly phosphorylated at DSBs in chromatin by the kinases ataxia-telangiectasia mutated (ATM) and ATM- and Rad3-related (ATR) (21). H2AX phosphorylation plays a key role in DNA damage checkpoint activation (21). VPA can enhance radiosensitivity, and this enhancement is correlated with histone hyperacetylation, chromatin decondensation, and enhanced formation of IRinduced $\mathrm{H} 2 \mathrm{AX}$ phosphorylation preferentially on euchromatic alleles rather than on heterochromatin (19). We found that phosphorylation of $\mathrm{H} 2 \mathrm{AX}$ was enhanced in retinoblastoma 
A
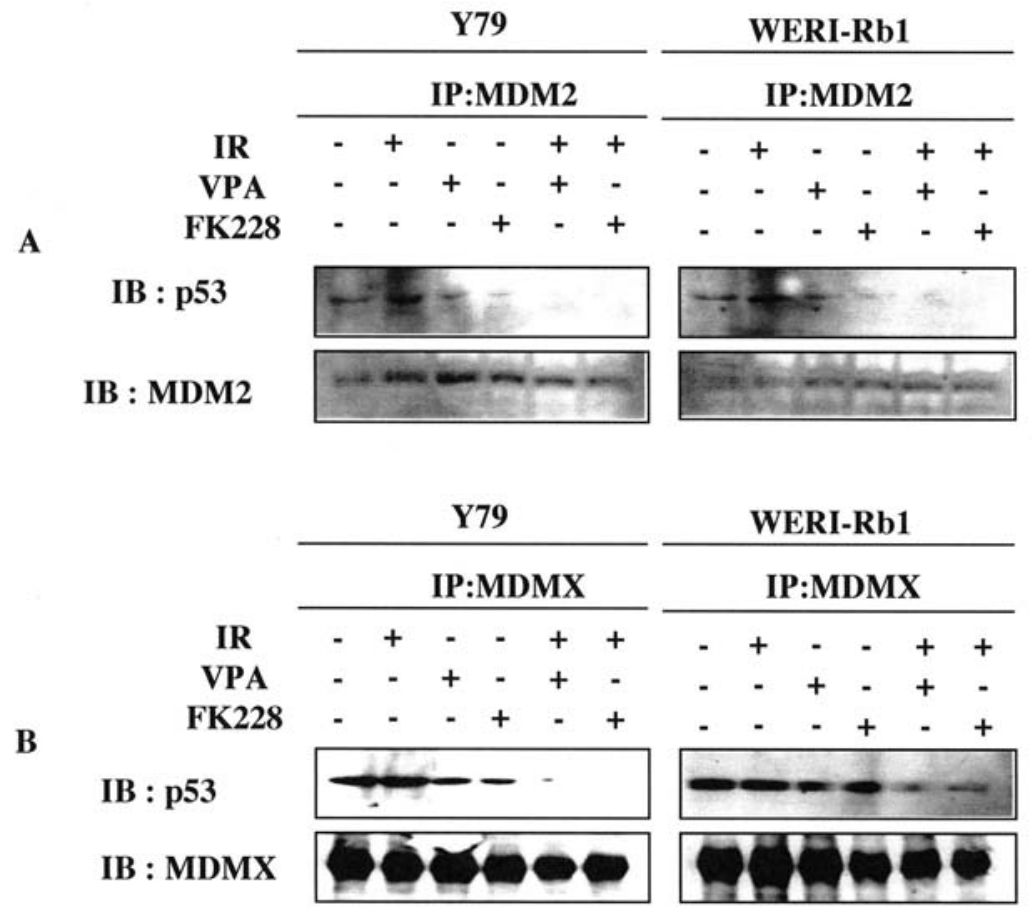

Figure 6. Effect of HDAC inhibitors on the interaction of MDM2/X and p53. Samples treated with both IR (2 Gy) and VPA ( 2 mM in Y79 cells, and $1 \mathrm{mM}$ in WERI-Rb1 cells) or both IR and FK228 ( $2 \mathrm{nM}$ in Y79 cells, and $1 \mathrm{nM}$ in WERI-Rb1 cells) for $48 \mathrm{~h}$ were immunoprecipitated for MDM2/X and then immunoblotted for $\mathrm{p} 53$ and MDM2 (A) or MDMX (B).
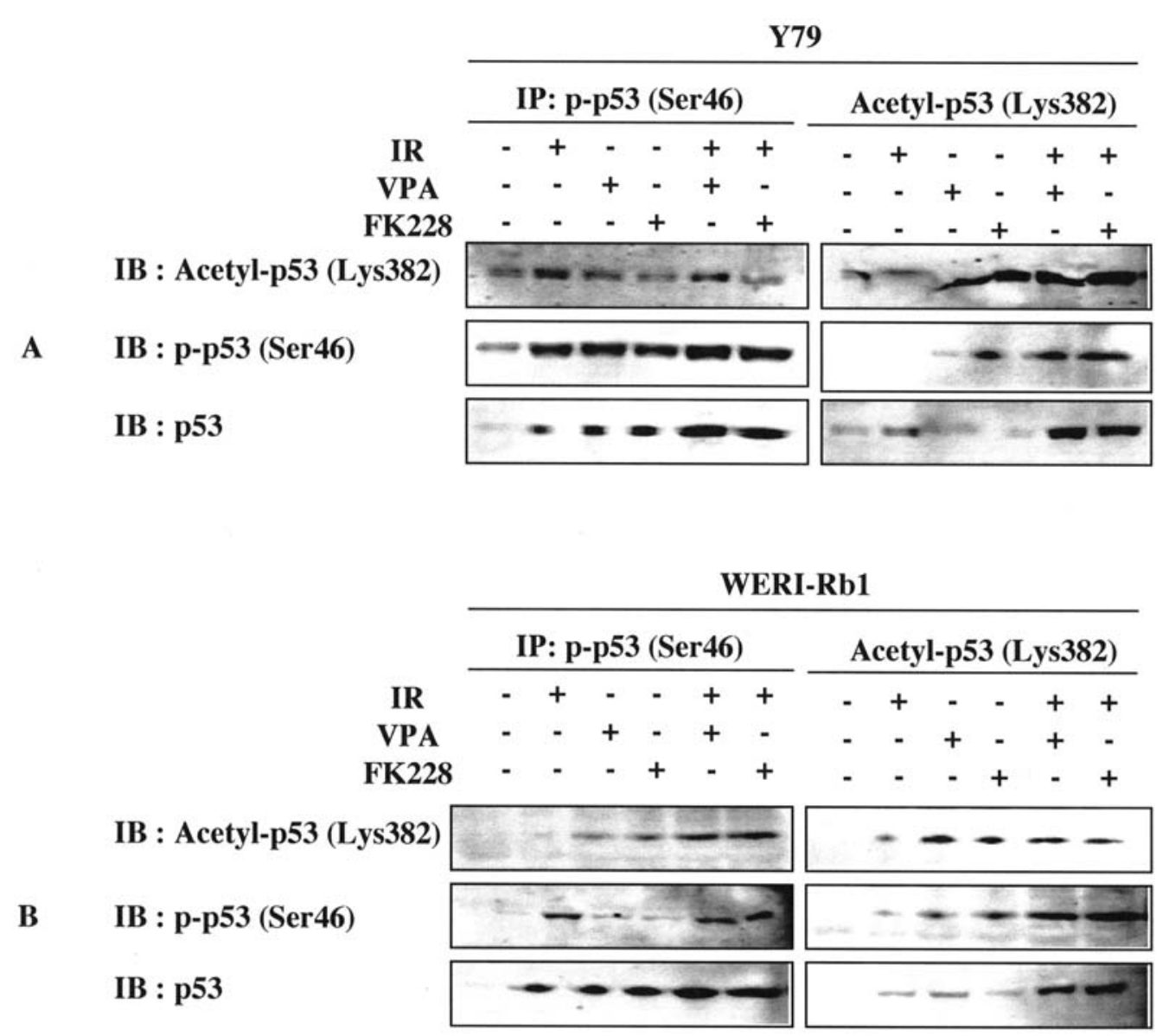

Figure 7. Effect of HDAC inhibitors on the acetylation of phosphorylated p53 (Ser46) in Y79 cells and WERI-Rb1 cells. Samples treated with both IR (2 Gy) and VPA ( $2 \mathrm{mM}$ in Y79 cells, and $1 \mathrm{mM}$ in WERI-Rb1 cells) or both IR and FK228 (2 nM in Y79 cells, and $1 \mathrm{nM}$ in WERI-Rb1 cells) for $48 \mathrm{~h}$ were immunoprecipitated for phosphorylated p53 (Ser46) or acetylated p53 (Lys382) and then immunoblotted for p53, acetylated p53 (Lys382), and phosphorylated p53 (Ser46) in Y79 cells (A) and WERI-Rb1 cells (B). 
cells treated with both IR and VPA or with both IR and FK228 rather than with IR, VPA, or FK228 alone. Consistent with our data, recent studies have shown that HDAC inhibitors prolong IR-induced phosphorylation of H2AX on Ser139, suggesting that HDAC inhibitors interfere with the repair of DSBs (17-19). H2AX adjacent to DSBs caused by IR is rapidly phosphorylated and may be repaired by the non-homologous end-joining (NHEJ) pathway or the homologous recombination pathway (18). SAHA has an inhibitory effect on NHEJ following IR exposure in A375 melanoma cells; this effect is associated with downregulation of $\mathrm{Ku} 70, \mathrm{Ku} 80$, and $\operatorname{Rad} 50$ (22). Moreover, treatment of cells with HDAC inhibitors, such as tricostatin A, SAHA, MS-275, and OSU-HDAC42, increases acetylation of $\mathrm{Ku} 70$, which was accompanied by reduced DNA-binding affinity (23).

In the present study, we demonstrated by means of IP experiments that VPA and FK228 reduce the IR-induced interaction of $\mathrm{p} 53$ with MDM2/X by acetylating phosphorylated p53 (Ser46) at Lys387 in Y79 and WERI-Rb1 cells. p53 protein has numerous sites for phosphorylation and acetylation and is activated by DNA damage through a phosphorylation-acetylation cascade $(24,25)$. The present results suggest 2 possible molecular mechanisms by which the HDAC inhibitors VPA and FK228 sensitize retinoblastoma cells to IR: i) enhancement of IR-induced DSBs and ii) acetylation of phosphorylated p53 leading to stabilization and accumulation of p53 through decreased binding to MDM2/X and, thereby, to apoptosis.

In addition, from the clinical standpoint, it is important to justify the concentrations of VPA and FK228 used in the present study. VPA is used as an antiepileptic drug, and its desired trough serum level for antiepileptic efficacy ranges from 50 to $100 \mu \mathrm{g} / \mathrm{ml}$, equivalent to $0.3-0.6 \mathrm{mM}$ (14). The peak concentration is observed $\sim 4 \mathrm{~h}$ after oral administration (26), and peak serum concentrations of VPA range from 110 to $1840 \mu \mathrm{g} / \mathrm{ml}$, with a mean of $378.3 \pm 310.2 \mu \mathrm{g} / \mathrm{ml}$ (27). Thus, the concentrations of VPA in the present study, 1-2 mM, seem to be clinically achievable. As for FK228, a phase I trial in patients with refractory neoplasms indicated a phase II dose of $17.8 \mathrm{mg} / \mathrm{m}^{2}$ administered by intravenous infusion on days 1 and 5 of a 21-day cycle, and the observed maximum plasma concentration was $553.8 \pm 299.5 \mathrm{ng} / \mathrm{ml}$, equivalent to $1.02 \pm 0.55 \mathrm{nM}$ (28). Therefore, the concentrations of FK228 used in the present study (2-10 $\mathrm{nM})$ also seem to be clinically achievable. The concentrations for antitumor efficacy may be achieved by local treatments, such as selective ophthalmic arterial injection, of these drugs.

Thus, HDAC inhibitors are expected to overcome the resistance of retinoblastoma to conventional eye-preserving therapy, to allow irradiation doses to be reduced, and to decrease rates of side effects, such as facial deformity, retinopathy, choroidopathy, optic neuropathy, and secondary neoplasms. Further study to clarify the molecular mechanism underlying the antitumor activity of VPA and FK228 may provide a novel rationale for the development of HDAC inhibitors for use in combination with conventional systemic/local chemotherapies or IR to treat malignant retinoblastoma.

\section{Acknowledgements}

This work was supported in part by grants from the Ministry of Education, Culture, Sports, Science and Technology of Japan (16790594) and Kawano Masanori Memorial Foundation for Promotion of Pediatrics (to M. Akiyama).

\section{References}

1. Shields CL, Meadows AT, Leahey AM and Shields JA: Continuing challenges in the management of retinoblastoma with chemotherapy. Retina 24: 849-862, 2004.

2. Yanagisawa T: Systemic chemotherapy as a new conservative treatment for intraocular retinoblastoma. Int J Clin Oncol 9: 13-24, 2004.

3. Suzuki S and Kaneko A: Management of intraocular retinoblastoma and ocular prognosis. Int J Clin Oncol 9: 1-6, 2004.

4. Hollstein M, Sidransky D, Vogelstein B and Harris CC: p53 mutations in human cancers. Science 253: 49-53, 1991.

5. Nork TM, Poulsen GL, Millecchia LL, Jantz RG and Nickells RW: p53 regulates apoptosis in human retinoblastoma. Cancer Res 115: 213-219, 1997.

6. Divan A, Lawry J, Dunsmore IR, Parsons MA and Royds JA: p53 and p $21^{\text {waf- }}$ expression correlates with apoptosis or cell survival in poorly differentiated, but not well-differentiated, retinoblastoma. Cancer Res 61: 3157-3163, 2001.

7. Appella E and Anderson CW: Post-translational modifications and activation of p53 by genotoxic stress. Eur J Biochem 268: 2764-2772, 2001

8. Shangary S and Wang S: Targeting the MDM2-p53 interaction for cancer therapy. Clin Cancer Res 14: 5318-5324, 2008

9. Marine JCW, Dyer MA and Jochemsen AG: MDMX: from bench to bedside. J Cell Sci 120: 371-378, 2007.

10. Wade $\mathrm{M}$ and Wahl GM: Targeting $\mathrm{mdm} 2$ and $\mathrm{mdmx}$ in cancer therapy: better living through medicinal chemistry? Mol Cancer Res 7: 1-11, 2009.

11. Laurie NA, Donovan SL, Shih CS, Zhang J, Mills N, Fuller C, Teunisse A, Lam S, Ramos Y, Mohan A, Johnson D, Wilson M, Galindo CR, Quarto M, Francoz S, Mendrysa SM, Guy RK, Marine JC, Jochemsen AG and Dyer MA: Inactivation of the p53 pathway in retinoblastoma. Nature 444: 61-66, 2006.

12. Monneret C: Histone deacetylase inhibitors. Eur J Med Chem 40: 1-13, 2005.

13. Phiel CJ, Zhang F, Huang EY, Guenther MG, Lazar MA and Klein PS: Histone deacetylase is a direct target of valproic acid, a potent anticonvulsant, mood stabilizer, and teratogen. J Biol Chem 276: 36734-36741, 2001.

14. Wolff JEA, Kramm C, Kortmann RD, Pietsch T, Rutkowwski S, Jorch N, Gnekow A and Driever PH: Valproic acid was well tolerated in heavily pretreated pediatric patients with high-grade glioma. J Neurooncol 90: 309-314, 2008.

15. Furumai R, Matsuyama A, Kobashi N, Lee KH, Nishiyama M, Nakajima H, Tanaka A, Komatsu Y, Nishino N, Yoshida M and Horinouchi S: FK228 (Depsipeptide) as a natural prodrug that inhibits class I histone deacetylases. Cancer Res 62: 4916-4921, 2002.

16. Konstantinopoulos PA, Vandoros GP and Papavassiliou AG: FK228 (depsipeptide): a HDAC inhibitor with pleiotropic antitumor activities. Cancer Chemother Pharmacol 58: 711-715, 2006.

17. Dalgard CL, Quill KRV and O'Brien JM: Evaluation of the in vivo antitumor activity of histone deacetylase inhibitors for the therapy of retinoblastoma. Clin Cancer Res 14: 3113-3123, 2008.

18. Shrivastav M, Haro LPD and Nickoloff JA: Regulation of DNA double-strand break repair pathway choice. Cell Res 18: 134-147, 2008.

19. Camphausen K, Burgan W, Cerre M, Oswald KA, Trepel JB, Lee MJ and Tofilon PJ: Enhanced radiation-induced cell killing and prolongation of $\gamma \mathrm{H} 2 \mathrm{AX}$ foci expression by the histone deacetylase inhibitor MS-275. Cancer Res 64: 316-321, 2004.

20. Zhang Y, Adachi M, Zou H, Hareyama M, Imai K and Shinomura Y: Histone deacetylase inhibitors enhance phosphorylation of histone $\mathrm{H} 2 \mathrm{AX}$ after ionizing radiation. Int J Radiat Oncol Biol Phys 65: 859-866, 2006.

21. Chinnaiyan P, Cerna D, Burgan WE, Beam K, Williams ES, Camphausen $\mathrm{K}$ and Tofilon PJ: Postradiation sensitization of the histone deacetylase inhibitor valproic acid. Clin Cancer Res 14: 5410-5415, 2008. 
22. Munshi A, Tanaka T, Hobbs ML, Tucker SL, Richon VM and Meyn RE: Vorinostat, a histone deacetylase inhibitor, enhances the response of human tumor cells to ionizing radiation through prolongation of $\gamma$-H2AX foci. Mol Cancer Ther 5: 1967-1974, 2006.

23. Chen CS, Wang YC, Yang HC, Huang PH, Kulp SK, Yang CC $\mathrm{Lu}$ YS, Matsuyama S, Chen CY and Chen CS: Histone deacetylase inhibitors sensitize prostate cancer cells to agents that produce DNA double-strand breaks by targeting Ku70 acetylation. Cancer Res 67: 5318-5327, 2007.

24. Bode AM and Dong Z: Post-translational modification of $\mathrm{p} 53$ in tumorigenesis. Nat Rev Cancer 4: 793-805, 2004.

25. Sakaguchi K, Herrera JE, Saito S, Miki T, Bustin M, Vassilev A, Anderson CW and Appella E: DNA damage activates p5 through a phosphorylation-acetylation cascade. Gene Dev 15: 2831-2841, 1998.
26. Vasudev K, Das S, Goswami U and Tayal G: Pharmacokinetics of valproic acid in patients with bipolar disorder. J Psychopharmacol 15: 187-190, 2001.

27. Spiller HA, Krenzelok EP, Klein-Schwartz W, Winter ML, Weber JA, Sollee DR, Bangh SA and Griffith JR: Multicenter case series of valproic acid ingestion: serum concentrations and toxicity. J Toxicol Clin Toxicol 38: 755-760, 2000.

28. Sandor V, Bakke S, Robey RW, Kang MH, Blagosklonny MV, Bender J, Brooks R, Piekarz RL, Tucker E, Figg WD, Chen KK, Goldspiel B, Fojo AT, Balcerzak SP and Bates SE: Phase I trial of the histone deacetylase inhibitor, depsipeptide (FR901228, NSC 630176), in patients with refractory neoplasms. Clin Cancer Res 8: 718-728, 2002. 